\title{
Collagen network remodelling and left ventricular function in constrictive pericarditis
}

Massimo Chello, Pasquale Mastroroberto, Rossana Romano, Francesco Perticone, Antonietta R Marchese

\begin{abstract}
Objective-To investigate whether patients with constrictive pericarditis have changes in collagen content and architecture that could influence left ventricular function.

Design-Cohort study.

Setting-University teaching hospital.

Patients-Biopsy specimens of myocardium from 13 patients admitted consecutively for treatment of chronic constrictive pericarditis were compared with normal heart tissue taken at necropsy from 15 patients free of cardiac disease.
\end{abstract}

Intervention-Pericardiectomy through median sternotomy. Biopsy specimens (4 or 5) were taken from the left ventricular free wall.

Main outcome measures-Biochemical and histological assessment of total collagen content, relative proportion of type $I$ and III collagen, and amount of orthogonal collagen fibre meshwork (crosshatching) in the left ventricular tissue.

Results-There was more collagen in the myocardium of patients with constrictive pericarditis than in controls when measured either biochemically by hydroxyproline content $(89.4$ v $50.4 \mathrm{mg} / \mathrm{g}$ dry weight) or by histological measurement of the collagen fraction of the myocardium $(2.4 \% v 7 \cdot 0 \%)$. Neither of these measurements, however, correlated with left ventricular ejection fraction, pulmonary wedge pressure, or right ventricular end diastolic pressure. The thickness of the fibrous trabeculae in the myocardium was, however, inversely related to both left ventricular ejection fraction $(r=$ $0 \cdot 76)$ and deceleration time $(r=-0 \cdot 68)$. Trabecular thickening was also related to NYHA class, with those in class III and IV carditis. ${ }^{1-3}$ In many patients, pericardiectomy corrects the haemodynamic abnormalities and produces dramatic clinical improvements. However, in a small but significant proportion of patients with constrictive pericarditis diastolic ventricular function is impaired despite operation and the extent of the pericardial resection. Operative, clinical, haemodynamic, and necropsy findings indicate that the impaired cardiac performance is usually secondary to myocardial dysfunction rather than to unrelieved constriction. ${ }^{45}$

The passive elastic properties of myocardium, and thereby mechanical functions of the heart, are influenced by myocardial collagen content and composition. ${ }^{6}$ The myocardium is an elastic network of myocytes enmeshed in a collagen matrix that connects the myocytes and the supporting coronary vasculature. Recent scanning electron microscopy studies in animals showed an intimate anatomical relation between muscle cells and connective tissue matrix, particularly with the arrangements of collagen fibres. ${ }^{7}$ The major structural proteins of the myocardial collagen matrix are fibrillar collagens of types I and III. ${ }^{89}$ Type I collagen is usually present as thick fibres with a tensile strength like that of steel. Therefore type I collagen determines the stiffness of the myocardium. Type III collagen forms fine reticular networks and is more distensible than type I collagen. Alterations in the functional and structural composition of this elastic network of contractile and non-contractile components cause corresponding changes in systolic and diastolic functions.

Myocardial fibrosis is usually detected histologically, and several biopsy studies ${ }^{10}$ and necropsy ${ }^{11}$ show that it is associated with left ventricular disease. Myocardial collagen content can also be measured biochemically as the content of protein containing hydroxyproline. ${ }^{12}$ We used both approaches, histological and biochemical, to assess the collagen content, the relative proportions and the architecture of the major collagen types in left ventricular tissue of 13 patients with constrictive pericarditis.

\section{Patients and methods}

PATIENTS

We studied 13 consecutive patients admitted to the Department of Cardiac Surgery of the Medical School of Catanzaro from April 1992 to October 1994 for treatment of constrictive pericarditis. Informed consent was obtained from all patients under a protocol approved by having the greatest thickening.

Conclusion-Changes in collagen content and architecture may contribute to impaired ventricular function in patients with chronic constrictive pericarditis.

(Heart 1996;75:184-189)

Keywords: collagen; left ventricular function; constrictive pericarditis

Consistently good late results have been reported in many large series of patients undergoing pericardiectomy for constrictive peri-

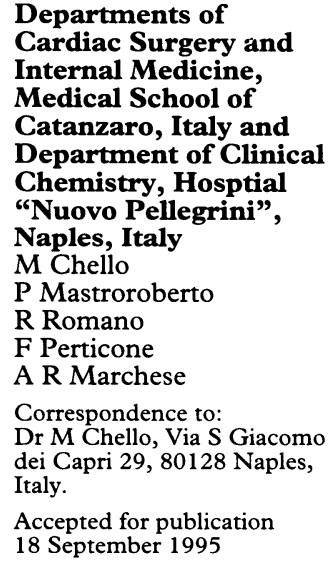


Table 1 Preoperative clinical characteristics

\begin{tabular}{|c|c|c|c|c|c|c|}
\hline Case & Age (yr) & Sex & Cause & $\begin{array}{l}\text { Duration of } \\
\text { symptoms (mnth) }\end{array}$ & $\begin{array}{l}\text { NYHA } \\
\text { functional class }\end{array}$ & $\begin{array}{l}\text { Pattern by } \\
\text { echo/Doppler }\end{array}$ \\
\hline 1 & 34 & $\mathbf{M}$ & Tuberculous & 5 & III & C \\
\hline 2 & 45 & $\mathbf{M}$ & Coxsackie B & 11 & III & $\mathrm{C}$ \\
\hline 3 & 52 & $\mathbf{F}$ & Unknown & 15 & II & $\mathrm{C}$ \\
\hline 4 & 49 & $\mathbf{M}$ & Staphylococcus & 18 & III & $\mathbf{R}$ \\
\hline 5 & 33 & $\mathrm{~F}$ & Tuberculous & 4 & III & $\mathrm{C}$ \\
\hline 6 & 64 & $\mathbf{M}$ & Unknown & 21 & IV & $\mathbf{R}$ \\
\hline 7 & 56 & M & Uraemic & 9 & II & C \\
\hline 8 & 47 & $\mathbf{M}$ & Tuberculous & 16 & III & $\mathrm{C}$ \\
\hline 9 & 60 & $\mathbf{F}$ & Unknown & 10 & III & $\mathrm{C}$ \\
\hline 10 & 37 & $\mathbf{F}$ & Unknown & 8 & III & $\mathrm{C}$ \\
\hline 11 & 54 & $\mathrm{M}$ & Unknown & 26 & IV & $\mathbf{R}$ \\
\hline 12 & 65 & $\mathbf{M}$ & Staphylococcus & 6 & III & C \\
\hline 13 & 40 & $\mathbf{M}$ & Uraemic & 3 & III & $\mathrm{C}$ \\
\hline
\end{tabular}

NYHA, New York Heart Association; C, constrictive; R, restrictive.

Table 2 Cardiac catheterisation data

\begin{tabular}{lllllllll}
\hline Patients & $\begin{array}{l}H R \\
\text { (beats/min) }\end{array}$ & $\begin{array}{l}M R A P \\
(\mathrm{~mm} \mathrm{Hg})\end{array}$ & $\begin{array}{l}M P A P \\
(\mathrm{~mm} \mathrm{Hg})\end{array}$ & $\begin{array}{l}R V E D P \\
(\mathrm{~mm} \mathrm{Hg})\end{array}$ & $\begin{array}{l}P W P \\
(\mathrm{~mm} \mathrm{Hg})\end{array}$ & $\begin{array}{l}L V E D P \\
(\mathrm{~mm} \mathrm{Hg})\end{array}$ & $\begin{array}{l}L V E F \\
(\%)\end{array}$ & $\begin{array}{l}C l \\
\left(l m i n \times m^{2}\right)\end{array}$ \\
\hline 1 & 95 & 25 & 29 & 25 & 27 & 27 & 60 & $2 \cdot 0$ \\
2 & 110 & 16 & 26 & 20 & 20 & 20 & 70 & $2 \cdot 7$ \\
3 & 124 & 17 & 25 & 21 & 21 & 23 & 60 & $2 \cdot 2$ \\
4 & 103 & 20 & 28 & 23 & 24 & 26 & 50 & $1 \cdot 8$ \\
5 & 90 & 23 & 27 & 25 & 24 & 25 & 50 & $1 \cdot 9$ \\
6 & 120 & 21 & 30 & 24 & 23 & 26 & 40 & $1 \cdot 7$ \\
7 & 88 & 15 & 26 & 21 & 18 & 19 & 65 & $2 \cdot 5$ \\
8 & 98 & 24 & 27 & 26 & 25 & 26 & 60 & $2 \cdot 2$ \\
9 & 105 & 20 & 24 & 23 & 21 & 22 & 50 & $2 \cdot 1$ \\
10 & 108 & 21 & 23 & 24 & 22 & 24 & 50 & $2 \cdot 1$ \\
11 & 120 & 23 & 29 & 24 & 25 & 26 & 40 & $1 \cdot 9$ \\
12 & 106 & 16 & 23 & 19 & 21 & 21 & 70 & $2 \cdot 3$ \\
13 & 100 & 19 & 25 & 22 & 22 & 24 & 50 & 2.1 \\
Mean & $105 \cdot 1$ & 20 & $26 \cdot 3$ & $22 \cdot 8$ & $22 \cdot 5$ & $23 \cdot 7$ & 55 & $2 \cdot 1$ \\
SD & $11 \cdot 3$ & $3 \cdot 2$ & $2 \cdot 2$ & $2 \cdot 1$ & $2 \cdot 4$ & $2 \cdot 5$ & 10 & $0 \cdot 2$ \\
\hline
\end{tabular}

$\mathrm{Cl}$, cardiac index; HR, heart rate; LVEDP, left ventricular end diastolic pressure; LVEF, left ventricular ejection fraction; MPAP, mean pulmonary artery pressure; MRAP, mean right atrial pressure; PWP, pulmonary wedge pressure; RVEDP, right ventricular end diastolic pressure.

the human studies committee of the university hospital. Table 1 shows the preoperative clinical characteristics.

Patients had had symptoms for 3-26 months (mean 11.6 months) before diagnosis; more than one third of patients had had them for more than a year. All patients had clinical evidence of pericardial constriction; the common physical signs of jugular venous distension $(77 \%)$, hepatomegaly $(70 \%)$, ascites $(61 \%)$, and peripheral oedema $(70 \%)$ were present in varying degree in all patients. A possible cause was identified in eight of 13 patients (table 1). In all patients the results of cardiac catheterisation were characteristic of a constrictive process (table 2). To exclude coronary narrowing as a cause of cardiac dysfunction coronary angiography was performed in all patients older than 40 years and in all with suspected coronary artery disease. No important coronary artery or valvar heart disease was found in any patient.

\section{ECHO-DOPPLER ASSESSMENT OF DIASTOLIC} FUNCTION

Two dimensional and $M$ mode echocardiograms were obtained with commercial cardiac

Table 3 Doppler velocity data in constrictive pattern (mean (SD))

\begin{tabular}{lcccc}
\hline & No & Apnoea & Inspiration & Expiration \\
\hline Mitral: & & & & \\
E (cm/s) & 10 & $67 \cdot 8(12)$ & $58 \cdot 6(10)$ & $79 \cdot 5(13 \cdot 2)$ \\
A (cm/s) & 10 & $57(10 \cdot 4)$ & $44 \cdot 2(8 \cdot 2)$ & $58 \cdot 7(11)$ \\
E/A & 10 & $1 \cdot 28(0 \cdot 24)$ & $1 \cdot 33(0 \cdot 12)$ & $1 \cdot 35(0 \cdot 2)$ \\
DT (ms) & 10 & $151 \cdot 4(23 \cdot 4)$ & $142 \cdot 9(20 \cdot 5)$ & $148 \cdot 3(22 \cdot 2)$ \\
IVRT & 10 & $61 \cdot 2(13 \cdot 1)$ & $67 \cdot 3(14 \cdot 4)$ & $56 \cdot 1(12 \cdot 6)$ \\
\hline
\end{tabular}

E, peak velocity of early rapid inflow; $A$, maximal velocity of late $L V$ inflow due to atrial contraction; DT, deceleration time; IVRT, isovolumic relaxation time. ultrasound instrument. All patients were studied within 12 hours of cardiac catheterisation, and 6-10 days and 6 months after surgery. After baseline recording of the $M$ mode image, the subjects underwent pulsed Doppler examination of the transmitral flow from the cardiac apex. A heat-sensitive nasal respirometer was used in all patients to record the phase of the respiration cycle. Constrictive or restrictive patterns were characterised as described by Oh et al. ${ }^{13}$ An $M$ mode pattern consistent with pericardial thickening was present in 10 patients. Ventricular chamber sizes were normal in all patients. Fractional shortening ranged from $24 \%$ to $46 \%$ (mean $35 \%$ ) in the patients with constrictive pericarditis and from $16 \%$ to $39 \%$ (mean $25 \%$ ) in the patients with a restrictive pattern. Left ventricular ejection fraction (LVEF) ranged from $40 \%$ to $70 \%$ (mean (SD) 55 (10)\%). Ten patients showed Doppler velocity data typical of constrictive physiology (table 3) whereas three patients had Doppler features typical of restrictive physiology (table 4).

\section{OPERATION}

Pericardiectomy was performed through a median sternotomy in all patients, with cardiopulmonary bypass (CPB) standby. All patients were monitored intraoperatively with indwelling radial artery lines and a Swan-Ganz catheter. Changes in central venous pressure (CVP) during the operation were calculated from the anaesthesia records. The extent of pericardiectomy varied. The pericardium over both ventricles was routinely excised, and the resection was extended at least to the phrenic nerve on both sides. The extent of further 
Table 4 Doppler velocity data in restrictive pattern (mean (SD))

\begin{tabular}{llccc}
\hline & No & Apnoea & Inspiration & Expiration \\
\hline Mitral: & & & & \\
E (cm/s) & 3 & $79 \cdot 6(15 \cdot 6)$ & $78 \cdot 3(15 \cdot 3)$ & $82(17 \cdot 7)$ \\
A (cm/s) & 3 & $36 \cdot 6(7)$ & $35 \cdot 3(6 \cdot 5)$ & $39(8 \cdot 1)$ \\
E/A & 3 & $1.9(0 \cdot 05)$ & $2 \cdot 2(0 \cdot 17)$ & $2(0 \cdot 2)$ \\
DT (ms) & 3 & $122 \cdot 6(11)$ & $120 \cdot 6(10)$ & $126 \cdot 6(12)$ \\
IVRT & 3 & $61(9)$ & $66 \cdot 3(8)$ & $62 \cdot 6(7 \cdot 5)$ \\
\hline
\end{tabular}

$E$, peak velocity of early rapid inflow; $A$, maximal velocity of late $L V$ inflow due to atrial contraction; DT, deceleration time; IVRT, isovolumic relaxation time.

resection of the pericardium overlying the atrium or the intrapericardial portion of the venae cavae, pulmonary veins, or great vessels depended on the degree of difficulty in developing an appropriate plane of dissection. Particular attention was directed to excising any constricting epicardial layers in those patients in whom intraoperative monitoring of CVP showed no decline in central pressure and in whom the epicardium was clearly abnormal.

ENDOMYOCARDIAL BIOPSY

Endomyocardial biopsies were performed for each patient at time of operation with a 1.5 $\mathrm{mm}$ Tru Cut needle. Four or five biopsy specimens were taken from identical places: the triangular area between the left anterior descending artery, second or third diagonal branch, and the apex of left ventricle. Two samples were immediately preserved in liquid nitrogen for biochemical examination. Fifteen hearts taken at necropsy from patients free of cardiac disease were studied. The mean (SD) age of the patients at the time of death was 45 (8) years. Five endomyocardial biopsy samples from the same area of the left ventricular free wall as described above were taken from each heart.

\section{BIOCHEMICAL COLLAGEN ASSAY}

The collagen content was determined in myocardial tissue as described by Stageman and Stadler ${ }^{14}$ after the hydroxyproline concentration was measured. The relative proportions of type I and III collagens were determined after $\mathrm{CNBr}$ cleavage as described by Laurent et al. ${ }^{15}$ The variation between duplicate determinations on the same sample was less than $5 \%$.

\section{HISTOLOGICAL EXAMINATION}

In each patient two or three biopsy samples were fixed in buffered formalin, embedded in paraffin blocks, and sectioned at a thickness of $5 \mu \mathrm{m}$. These sections were stained with the picrosirius red technique for collagen ${ }^{16}$ and observed with a polarising microscope. Total collagen volume fraction (TCVF) and endocardial fibrosis were determined as described by Villari et al. ${ }^{17}$ The extent and thickness of orthogonal collagen fibre meshwork (cross hatching) were evaluated by the picrosiriuspolarisation technique and graded as described by Villari et al as $0=$ no cross hatching, $0.5=$ minimal, $1.0=$ moderate, $1.5=$ fair, 2 = extensive, and $3=$ massive cross hatching. Repeated measurements were performed on each section (variation $<5 \%$ ).
STATISTICAL ANALYSIS

The values presented are mean (1 SD). Comparisons between the two groups were performed by the unpaired Student $t$ test. We used linear regression to analyse relations between structural and haemodynamic data. A significance level of $<0.05$ was used.

\section{Results}

There were no operative deaths. At operation, seven patients $(54 \%)$ had parietal constriction only and six (46\%) had visceroparietal constriction. In the patients with visceroparietal constriction epicardial decortication was necessary. The only postoperative complication was low cardiac output state in four patients $(30 \%)$. Two of these patients were in functional class III and two were in class IV before operation. Both patients in class IV and one in class III had visceroparietal constriction. Intraoperative bleeding occurred in two patients as a result of laceration of the right ventricle when the sternotomy was performed. Of these patients, one required CPB to control bleeding. A significant decrease of CVP occurred in seven patients in the operating room soon after pericardiectomy, and in three patients within 48 hours of the operation. CVP did not change much in three patients, despite complete pericardiectomy and epicardial decortication.

All patients were followed for up to 18 months (median 10 months). One patient died within four months of operation because of progressive congestive heart failure. This patient, who underwent extensive pericardiectomy and epicardial decortication, had little improvement in cardiac function after pericardiectomy. The postoperative course was complicated by low cardiac output state requiring infusion of dopamine for 36 hours. Another patient, who had had considerable symptomatic improvement after pericardiectomy, remained free of cardiac symptoms until his later death from lung cancer.

After pericardiectomy, the considerable respiratory variation in mitral flow velocity and left ventricular relaxation time was no longer present in 10 patients. Of these, nine showed a preoperative constrictive Doppler pattern, and one a preoperative restrictive pattern. One patient with a preoperative constrictive

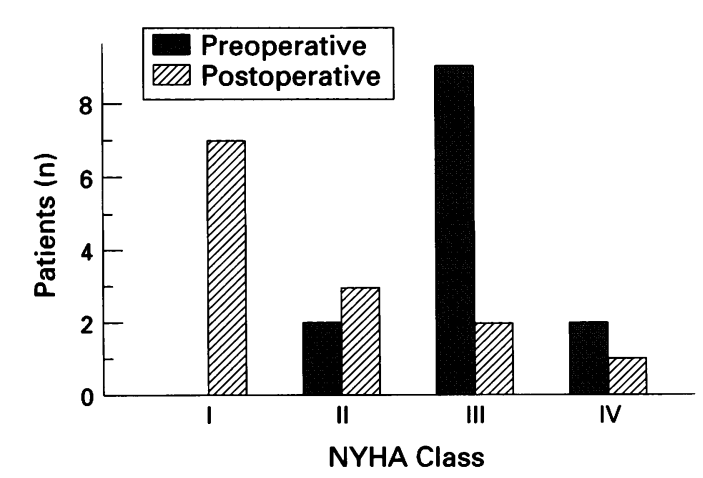

Figure 1 Preoperative and postoperative (follow up) functional state (New York Heart Association (NYHA)). 
Table 5 Collagen concentration and the relative proportion of types I and III in normal and diseased left ventricles (mean (SD))

DW, dry weight.

\begin{tabular}{llll}
\hline & Control & Pericarditis & $P$ \\
\hline Total collagen (mg/g DW) & $50 \cdot 4(20)$ & $89 \cdot 4(25)$ & $<0 \cdot 01$ \\
\% Type III & $40 \cdot 2(9)$ & $34 \cdot 3(7 \cdot 3)$ & $0 \cdot 07$ \\
Type III (mg/g DW) & $19 \cdot 2(6)$ & $30(9 \cdot 7)$ & $<0 \cdot 01$ \\
\% Type I & $59 \cdot 6(9 \cdot 6)$ & $64 \cdot 8(8 \cdot 5)$ & $0 \cdot 15$ \\
Type I (mg/g DW) & $31 \cdot 2(2 \cdot 8)$ & $59(5)$ & $<0 \cdot 01$ \\
\hline
\end{tabular}

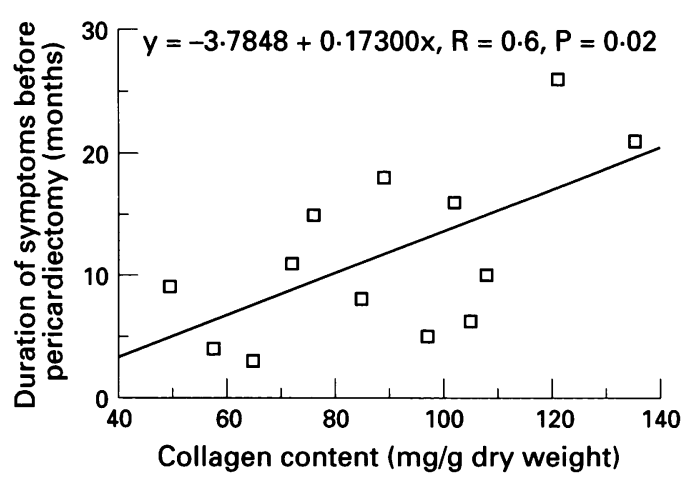

Doppler pattern developed a restrictive pattern after pericardiectomy without an intervening cardiac event. Two of three patients with a preoperative restrictive pattern still had symptoms (NYHA class III) at postoperative Doppler examination. Follow up showed an excellent result in 10 patients and a poor result in three patients (fig 1 ).

\section{BIOCHEMICAL ASSAY}

The collagen concentration in the left ventricle of patients with constrictive pericarditis was significantly higher than that in control ventricles $(P<0.01$, table 5$)$. Table 5 shows that the proportion of type III (compared with type I plus type III) was lower in patients with constrictive pericarditis than in controls $(P<$ $0.05)$. The estimated concentration of types I and III collagen, derived from the total collagen concentration and the proportion of each type, is also shown in table 5. There was a rise in both type I and III collagens, but there was a disproportional increase in type I.

\section{HISTOLOGICAL EXAMINATION}

Biopsy samples from hearts with constrictive pericarditis were more fibrotic than those from autopsy hearts (table 6). A trend toward a higher fibrosis was observed in patients with a restrictive echo pattern, even though not significant. The fibrous content varied widely between different samples. The fibrosis was subepicardial in three patients. In two cases the myocardial fibrosis was subendocardial or located in the zone intermediate between subendocardium and subepicardium; a generalised fibrosis was observed in three patients. An extensive collagen meshwork, as indicated by the cross hatching index of fibrils, was found in $46 \%$ of patients with constrictive pericarditis, compared with a minimal level in the control group.

\section{CORRELATION BETWEEN HAEMODYNAMIC AND} STRUCTURAL DATA

The aetiological subgroup was not related to the incidence of low cardiac output. A significant trend was observed between the interval between the onset of symptoms and total collagen content $(r=0.61, \mathrm{P}=0.02$, fig 2$)$. There was no correlation between myocardial colla-

Table 6 Histological quantitative data (mean (ISD)) in normal and diseased left ventricles

\begin{tabular}{llll}
\hline & Control & Pericarditis & $P$ \\
\hline TCVF (\%) & $2.4(0.9)$ & $7 \cdot 0(1)$ & $<0.001$ \\
Endocardial fibrosis (\%) & 0 & $6.8(4 \cdot 2)$ & $<0.001$ \\
Cross hatching grade & $0.4(0 \cdot 1)$ & $1.5(0.8)$ & $<0.01$ \\
\hline
\end{tabular}

$\mathrm{TCVF}$, total collagen volume fraction.
Figure 2 Correlation between duration of symptoms before pericardiectomy and total collagen content $(\mathrm{mg} / \mathrm{g} d \mathrm{dr} y$ weight). One point represents one patient.

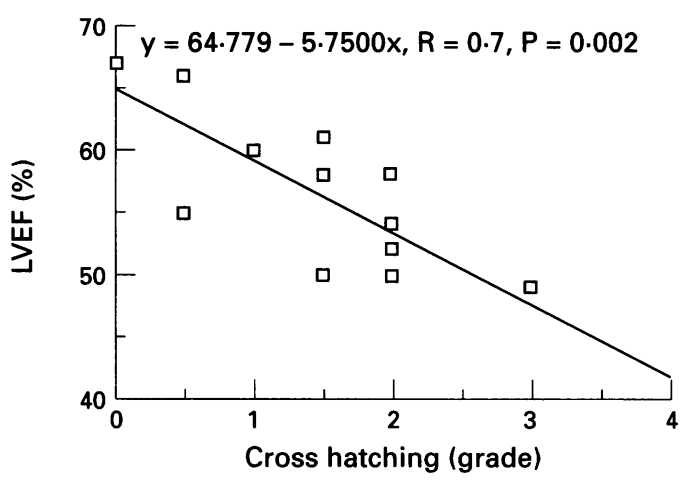

Figure 3 Correlation between cross hatching grade and left ventricular ejection fraction (LVEF). One point

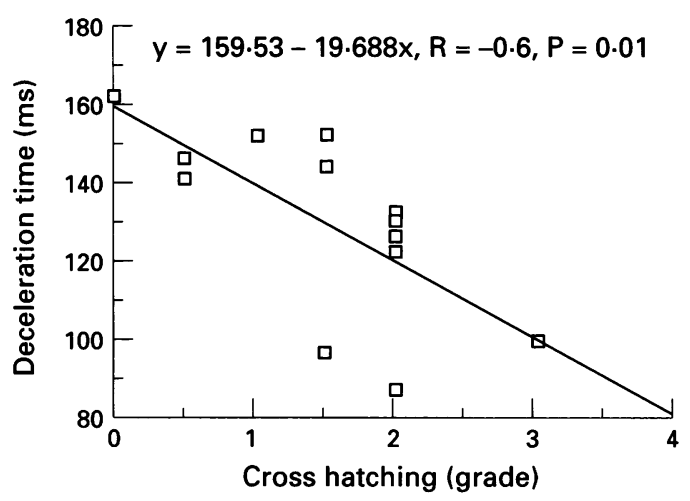

Figure 4 Correlation between cross hatching grade and deceleration time. One point represents one patient.

gen content and age. Linear regression analysis did not show any correlation between total collagen content and mean right atrial pressure, pulmonary wedge pressure, and right ventricular end diastolic pressure (RVEDP). Neither cardiac index (CI) nor LVEF correlated with total collagen content. There was a weak but significant correlation between the percentage of type I collagen and RVEDP ( $r=$ $0 \cdot 5, P=0.04)$. The extent of cross hatching was inversely correlated with both LVEF ( $r=$ $-0.76, P=0.002$, fig 3 ) and deceleration time $(r=-0 \cdot 68, \mathrm{P}=0.01$, fig 4$)$.

The measurement of fibrosis varied according to postoperative NYHA class. The grade of cross hatching was higher in patients in functional class III or IV $(n=3)$ than in patients in class $I(\mathrm{n}=7)(2.3(0.5) \quad v \quad 0.9$ $(0 \cdot 7), P=0 \cdot 03)$. represents one patient. 


\section{Discussion}

Although pericardiectomy is usually followed by an immediate and adequate relief of symptoms in constrictive pericarditis, ${ }^{1-318}$ sometimes there is no response after extensive pericardiectomy despite complete ventricular decortication. ${ }^{1920}$ Operative, clinic, haemodynamic, and necropsy findings indicate that impaired cardiac performance in these patients is usually secondary to myocardial dysfunction rather than to unrelieved constriction..$^{21} 22$ The natural course of continued postoperative deterioration in some patients with constrictive pericarditis accords with the possibility that restriction may not be limited to pericardial thickening, and myocardial dysfunction can result from myocardial involvement in the disease process that led to the constrictive pericarditis. ${ }^{23} 24$

The coexistence of pericardial constriction with myocarditis, postradiation cardiomyopathy, or other forms of cardiomyopathy has been described in a subset of patients reported by Seifert and coworkers. ${ }^{25}$ They found that outcome in patients with constrictive pericarditis and restrictive cardiomyopathy was no better than those with restrictive cardiomyopathy alone and were hesitant to recommend pericardial stripping in such patients, particularly the postradiation group. Levine reported on seven necropsy cases in patients operated on for constrictive pericarditis: there was severe myocardial fibrosis in four. ${ }^{22}$ Talwar et al performed endomyocardial biopsy in six patients with constrictive pericarditis, and found myocarditis and myocardial interstitial fibrosis in all irrespective of the endocardial thickening. ${ }^{26}$

Changes in collagen subtypes have already been reported in myocardial disease. An increase in the amount of type I collagen was recently described in the formation of scars after myocardial infarction ${ }^{27}$ and in cardiomyopathy and ischaemic heart disease. ${ }^{28}$ In the present study we showed a significant increase in collagen concentration and in the proportion of type I collagen compared with type III collagen, as well as altered arrangement of the newly synthesised collagen fibrils.

Nevertheless, there is still disagreement about whether or not there is a relation between increased collagen content and stiffness. Many experiments ${ }^{29}$ counter the idea that a decline in compliance in hypertrophied myocardium is related to a higher collagen content. The change in flexibility of the left ventricle could also be related to organisation of newly synthesised collagen fibrils or to alteration in its structure such as a greater degree of cross linking.

Recently, several studies in laboratory animals have shown that the structural characteristics and the arrangement of collagen fibres with respect to myocytes, and not solely the amount of collagen, influence the mechanical behaviour of the myocardium. ${ }^{30-34}$ In rats with pressure overload hypertrophy Jalil et al showed that systolic and diastolic stiffness were both altered in the presence of pathological realignment of collagen and muscle fibres. ${ }^{32} 33$
In 32 patients with aortic valve disease, Villari and coworkers found changes in collagen architecture associated with altered systolic function and passive diastolic properties. ${ }^{17}$ The isolated increase in total collagen volume fraction without a change in architecture leaves systolic and passive diastolic function unaltered. They also found that in the presence of myocardial fibrosis, ejection fraction was depressed and passive myocardial stiffness increased-further evidence that the endocardium modulates the performance of the adjacent myocardium. How fibroblasts are stimulated to produce more collagen of any type or to degrade it and what part the myocytes and the other components of the extracellular matrix play in this process are still unresolved questions. As Levine suggested, at least two different mechanisms could produce concomitant involvement of pericardium and myocardium:

- Subepicardial penetration by a primary pericardial inflammatory process. There is no rigid barrier at the epicardial membrane to prevent the intramyocardial extension of a bacterial or viral process that arises in the pericardium. On healing, the inflammatory myocarditis is commonly replaced by fibrous connective tissue.

- Impairment of coronary blood flow. Some have speculated that coronary blood flow may be altered by low blood pressure, ${ }^{35}$ the direct or indirect effects of compression resulting in increased external force upon the larger blood vessels, ${ }^{36}$ and by unusual transmural pressures affecting the smaller intramural coronary arteries. Another explanation of this may lie in capillary endothelial cell injury and proliferation of fibrous tissue leading to endoluminal narrowing and, ultimately, to myocardial ischaemia. ${ }^{37}$ It is possible that substantially diminished blood flow in many areas of the ventricle may lead to hypoxia and myocardial anaerobiosis with lactate production in the myocardium that could lead to excess collagen growth relative to muscle because of fibroblast stimulation. ${ }^{38}$

In conclusion, our study shows that in chronic constrictive pericarditis a significant increase of total collagen as well as an enhanced fibre meshwork and endocardial fibrosis are associated with altered systolic and diastolic myocardial properties. Because of the significant correlation between total collagen content and duration of symptoms before pericardiectomy, in patients who had had symptoms lasting for many months it would be advisable to perform a biopsy of the myocardium before pericardiectomy. The finding of advanced interstitial heart disease should be regarded as a useful predictor of the late postoperative functional outcome.

1 McCaughan BC, Hartzell V, Schaff HV, Piehler JM, Danielson GK, Orsulak TA, et al. Early and late results of pericardiectomy for constrictive pericarditis. $\mathcal{F}$ Thorac of pericardiectomy for constrictive
Cardiovasc Surg 1985;89:340-50.

2 De Valeria PA, Baumgartner WA, Casale AS, Greene PS, Cameron DE, Gardner TJ, et al. Current indications, risks, and outcome after pericardiectomy. Ann Thorac Surg 1991;52:219-24. 
3 Miller II, Mansour KA, Hatcher CR. Pericardiectomy: current indications, concepts, and results in a university cenrent indications, concepts, and result
ter. Ann Thorac Surg 1982;34:40-5.

ter. Ann Thorac Surg $1982 ; 34: 40-5$.
4 Viola AR. The influence of pericardiectomy on the hemodynamics of chronic constrictive pericarditis. Circulation 1973;48:1038-42.

5 Kloster FE, Crislip RL, Bristow JD, Herr RH, Ritzman LW, Griswold HE. Hemodynamic studies following pericardiectomy for constrictive pericarditis. Circulation 1965;32:415-24

6 Thiedemann KU, Houbarsch CH, Medugoral I, Jacob R Connective tissue content and myocardial stiffness in pressure overload hypertrophy. A combined study of morphologic, morphometric, biochemical and

7 Caulfield JB, Borg TK. The collagen network of the heart. Lab Invest 1979;40:364-72.

8 Medugoral I. Characterization of intramuscular collagen in the mammalian left ventricle. Bas Res Cardiol 1982;77 589-98.

9 Miller EJ. Biochemical characteristics and biological significance of the genetically distinct collagens. Mol Cell

10 Oldershaw PJ, Brooksby IAB, Davies MJ, Coltart DJ, Jenkins BS, Webb-Peploe MM. Correlations of fibrosis in endomyocardial biopsies from patients with aortic valve disease. Br Heart f 1980;44:609-11.

11 Anderson KR, St John Sutton MG, Lie JT. Histopathological types of cardiac fibrosis in myocardial disease. $f$ Pathol 1979;128:79-85.

12 Frederiksen DW, Hoffnung JM, Frederiksen RT, Williams RB. The structural proteins of normal and diseased human myocardium. Circ Res 1978;42:459-66.

13 Oh JK, Hatle LK, Seward JB, Danielson GK, Schaff HV, Reeder GS, et al. Diagnostic role of echocardiography in
constrictive pericarditis. $\mathcal{F}$ Am Coll Cardiol 1994;23: 154-62.

14 Stageman H, Stadler K. Determination of hydroxyproline. Clin Chim Acta 1967;18:267-73.

15 Laurent GJ, Cockerill P, McAnulty RJ, Hastings JRB. A simplified method for quantitation of the relative amounts of type I and type III collagen in small tissue amounts of type I and type III collagen

16 Junqueira LCU, Cossermelli W, Brentani R. Differential staining of collagens type I, II and III by sirius red polarstaining of collagens type I, II and III by sirius red polar

17 Villari B, Campbell SE, Hess OM. Influence of collagen network on left ventricular systolic and diastolic function in aortic valve disease. $7 \mathrm{Am}$ Coll Cardiol 1993;22. $1477-84$

18 Stalpaert G, Suy R, Daenen W, Nhevelsteen A. Total pericardiectomy for constricting pericarditis. Early and late results. Acta Chir Belg 1981;5:277-82.

19 Sommerville W. Constrictive pericarditis with special reference to the change in natural history brought about by surgical intervention. Circulation 1968;38(suppl 5): 102-11.

20 Collins HA, Woods LP, Daniel RA. Late results of pericardiectomy. Chronic constrictive pericarditis. Arch Surg 1964;89:921-28.
21 Meaney E, Shabetai R, Bhargava V, Shearer M, Weidner C, Manglardi LM, et al. Cardiac amyloidosis, constrictive pericarditis, and restrictive cardiomyopathy. Am 7 Cardiol pericarditis, and rest

22 Levine HO. Myocardial fibrosis in constrictive pericarditis. Electrocardiographic and pathologic observations. Circulation 1973;48:1268-81.

23 Dines DE, Edwards JE, Burchell JB. Myocardial atrophy in constrictive pericarditis. Mayo Clin Proc 1958;33:93-99.

24 Roberts JT, Beck CS. The effect of chronic cardiac compression on the size of the heart human fibers. Am Heart $\mathcal{f}$ 1941;22:314-20.

25 Seifert FC, Miller DC, Oesterle SN, Oyer PE, Stinson EB, Shumway NE. Surgical treatment of constrictive pericarditis: analysis of outcome and diagnostic error. Circulation 1985;72(suppl II):264-73.

26 Talwar KK, Narula JP, Chopra P. Myocarditis and myocardial interstitial fibrosis in constrictive pericarditis - an extended pathological spectrum? Int $\mathcal{f}$ Cardiol 1990;29:241-43.

27 Whittaker P, Boughner D, Kloner R. Analysis of healing after myocardial infarction using polarised light microscopy. Am $₹$ Path 1989;134:879-93.

28 Greenshop J, Greenbaum R, Gibson D, Yacoub M, Laurent G. Enhanced deposition of predominantly type I collagen in myocardial disease. $\mathcal{F} \mathrm{Mol}$ Cell Cardiol $1990 ; 22: 1157-65$.

29 Badeer HS. Development of cardiomegaly: a unifying hypothesis explaining the growth of muscle fibers, blood vessels and collagen of heart. Cardiology 1972;57:247-54.

30 Weber KT. Cardiac interstitium in health and disease: the fibrillar collagen network. $₹ \mathrm{Am}$ Coll Cardiol 1989;3: 1637-52.

31 Weber KT, Brilla CG. Pathological hypertrophy and cardiac interstitium. Circulation 1991;83:1849-65.

32 Jalil JE, Janicki JS, Pick R, Abrahams C, Weber KT. Fibrosis induced reduction of the endomyocardium in the rat after isoproterenol treatment. Circ Res 1989;65: 258-64.

33 Jalil JE, Doering CW, Janicki JS, Pick R, Shroff SG, Weber KT. Fibrillar collagen and myocardial stiffness in the intact hypertrophied rat left ventricle. Circ Res 1989;64: intact hypert

34 Weber KT, Pick R, Janin JE, Janicki JS, Carrol EP. Patterns of myocardial fibrosis. $尹$ Mol Cell Cardiol 1989; 21(suppl V):121-31

35 Spodick DH. Chronic and constrictive pericarditis. New York: Grune and Stratton 1964:134-8.

36 Braunwal E, Sarnoff SJ, Case RB, Stainsby WN, Welch $\mathrm{CH}$ Jr. Hemodynamic determinants of coronary flow: effects of changes in aortic pressure and cardiac output and the relationship between myocardial oxygen consumption and coronary flow. Am f Physiol 1958;192: 157-63.

37 Stewart JR, Fajardo LF. Radiation-induced heart disease. Clinical and experimental aspects. Radiol Clin North Am 1971;9:511-31.

38 Comstock JP, Underfrind S. Effect of lactate on collagen proline hydroxylase activity in cultured L-929 fibroblasts. Proc Natl Acad Sci 1970;66:552-59. 